\title{
Bonding durability of dental sealants to deciduous and permanent teeth
}

\author{
Sandra Kiss Moura ${ }^{1}$, Letícia Vargas Freire Martins Lemos², Suellen Myszkovisk', \\ Maria Gisette Arias Provenzano ${ }^{1}$, Ivan Balducci ${ }^{3}$, Silvio Issao Myaki ${ }^{2}$ \\ ${ }^{1}$ Universidade Norte do Paraná - UNOPAR, School of Dentistry, Department of Restorative Dentistry, Londrina, PR, Brazil \\ 2Universidade Estadual Paulista - UNESP, School of Dentistry, Department of Paediatric Dentistry, São José dos Campos, SP, Brazil \\ ${ }^{3}$ Universidade Estadual Paulista - UNESP, School of Dentistry, Department of Mathematics and Statistics, São José dos Campos, SP, Brazil
}

\begin{abstract}
Aim: To evaluate the bonding durability of materials used as sealants on different dentitions. Methods: Deciduous (D) and permanent (P) molars were divided into four groups $(n=5)$ and sealed with Fluroshield $(\mathrm{F})$ and OptiBond FL (FL). Blocks of composite resin were built, stored in distilled water $\left(24 \mathrm{~h} / 37^{\circ} \mathrm{C}\right)$, sectioned into $0.8 \mathrm{~mm}^{2}$ and tested at tensile $(0.5 \mathrm{~mm} / \mathrm{min})$ after $24 \mathrm{~h}$ and 6 months. The fractures were observed and classified into adhesive, cohesive or mixed types. The data were analyzed using repeated measures ANOVA and Tukey test $(\alpha=5 \%)$, with tooth as the experimental unit. Results: There were differences for dentition ( $p=0.0097)$, dental sealant $(p=0.0019)$ and time $(p=0.0001)$. At $24 \mathrm{~h}$ the highest bond strength was observed for OptiBond FL on deciduous and permanent teeth, similar to Fluroshield at both dentitions. After 6 months the bond strength of OptiBond FL did not decrease in the permanent teeth, but decreased similar to Fluroshield in deciduous teeth, the bond strength of Fluroshield decreased similarly in both dentitions. Conclusions: The bond strength of dental sealants varied with the experimental conditions.
\end{abstract}

Keywords: dental enamel; longevity; pit and fissure sealants.

\section{Introduction}

The enamel of deciduous and permanent teeth differs in terms of chemical composition, morphology and physiology ${ }^{1-4}$. Deciduous teeth have thinner enamel ${ }^{2}$ and thicker aprismatic layer ${ }^{2-3}$. In addition, they are more susceptible to dental caries and erosion, and the bonding of dental materials may vary from that of permanent teeth ${ }^{5-6}$. It is also known that $81.5 \%$ of dental caries found in children 6-36 months of age occur in the posterior teeth ${ }^{7}$.

A recent study ${ }^{8}$ has shown that pain and dental caries in the primary dentition

Received for publication: May 18, 2014 Accepted: August 25, 2014

Correspondence to: Sandra Kiss Moura Universidade Norte do Paraná (UNOPAR) Curso de Odontologia Rua Marselha, 183 - Jardim Piza CEP 86041-140 - Londrina, Paraná, Brasil Phone: +55 4333717820 - Fax: +55 4333418122 E-mail: kissmoura@gmail.com were the main reasons to visit dentists and the relationship between dental caries in deciduous teeth is a predictor for the disease in permanent dentition. Morphology of the occlusal surface favors the accumulation of biofilm and increases the risk for development of dental caries, as shown in a study with children between 2 and 3 years old, in which $74.5 \%$ of them had dental caries on the occlusal surface 9 .

Dental sealants act as a mechanical barrier to the accumulation of biofilm, minimizing the risk for development of dental caries ${ }^{10}$. However, application of some types of sealants may be complicated in children, due to the risk of saliva contamination during the procedure. Sealants are traditionally hydrophobic and hydrophilic materials investigated as an alternative to overcome the challenges 
in sealant application ${ }^{11-14}$. Difference in formulations of dental materials may have an impact on the bonding ability of dental sealants to deciduous and permanent teeth. This would be relevant to understand the importance of testing different materials for pit and fissure sealants ${ }^{15-16}$.

A previous study has shown superior adhesion of OptiBond FL compared with a traditional hydrophobic sealant used in pediatric dentistry, $24 \mathrm{~h}$ after being applied to the occlusal surface of molars ${ }^{17}$. Other studies have confirmed the feasibility of using certain sealant adhesive systems on occlusal surfaces in pediatric dentistry ${ }^{12-13}$. However, there are few studies that assess the durability of this procedure, especially comparing the deciduous and permanent dentitions.

The efficacy of sealants is related to their retention to enamel ${ }^{18}$. Since deciduous and permanent teeth have different properties, it is important to test different sealant materials in both dentitions. Therefore, the aim of this study was to evaluate the microtensile bond strength of sealant materials to the enamel of primary and permanent teeth, after $24 \mathrm{~h}$ and 6 months. The tested hypothesis is that the microtensile bond strength of different dental sealants to deciduous and permanent tooth does not vary along the time.

\section{Material and methods}

Ten deciduous molars obtained no longer than 6 months after exfoliation ${ }^{13}$ were disinfected in $0.5 \%$ chloramine solution at $4{ }^{\circ} \mathrm{C}$ and cleaned with pumice and water slurry. The same procedure was used with 10 extracted, caries-free third permanent molars. All teeth were used for bond strength measurement. The research protocol was approved by the local Ethics Committee (Protocol PP0096/11).
The deciduous and permanent teeth were randomly assigned to four groups $(n=5)$, according to the type of dentition and dental sealant: Permanent OptiBond FL, Permanent Fluroshield, Deciduous OptiBond FL and Deciduous Fluroshield. The occlusal surfaces of all teeth were cleaned with pumice and water prior to sealing. The sealants were a hydrophobic pit-and-fissure sealant (Fluroshield; Dentsply, Petrópolis, RJ, Brazil) and a conventional adhesive system (OptiBond FL; Kerr Co, Orange, CA, USA). A trained operator performed all sealing procedures in accordance with the manufacturers' recommendations (Table 1).

The pulp chamber of each primary tooth was restored to prevent enamel cracking during preparation of the specimens for the microtensile bond strength test ${ }^{13}$. This procedure is required because after exfoliation only a thin layer of dentin remains in the crown. The pulp chambers of 10 primary molars were acid-etched with $37.5 \%$ phosphoric acid for $15 \mathrm{~s}$, washed for $15 \mathrm{~s}$, dried, and restored with Adper Single Bond 2 adhesive system and Filtek Z250 composite (shade A3,5; 3M ESPE Dental Products; St Paul, MN, USA), according to the manufacturer's directions (Table 1). After application, the restoring materials were photoactivated with light intensity of $600 \mathrm{~mW} / \mathrm{cm}^{2}$ (VIP; Bisco, Schaumburg, IL, USA). The sealants were placed on the occlusal surfaces in a single increment and according to the manufacturer's instructions. In sequence, Filtek Z250 resin blocks (3M ESPE Dental Products, St Paul, MN, USA) were built up in three 2mm-increments on sealant surface.

After storage in distilled water for $24 \mathrm{~h}$ at $37{ }^{\circ} \mathrm{C}$, the restored teeth were serially sectioned ${ }^{12-13}$ with a diamond disk (Extec 12205 High Concentration; Enfield, CT, USA) in a

Table 1 - Used materials, composition and application modes

\section{Material}

(Batch number)

OptiBond FL

(Primer 3124126;

Adhesive 3101101)

uroshiel

(047065A)

Dental Gel Etchant: phosphoric acid, water, colloidal silica, inorganic colorFluroshield (50\% inorganic fillers): Bis-GMA modified urethane, TEGDMA, aluminum and barium borosilicate, phosphoric acid tetracyclic ester, sodium fluoride, n-methyldiethanolamine, camphoroquinone.

Filtek Z250

(6BR)

Adper Single Bond 2

(8RL)

\section{Composition}

Primer: $37.5 \%$ phosphoric acid; Ethyl alcohol (20-25\%), alkyl dimethacrylate resins, water; Adhesive: uncured methacrylate Ester (50-60\%), TEGDMA (5-10\%), Ytterbium trifluoride (12-17\%), inert mineral fillers, photoinitiators, stabilizers

\section{Application Mode}

1- Acid etching (15 s); 2- Wash (15 s);3- Dry (5 s); 4- Apply one coat of primer (15 s); 5- Dry (5 s at $10 \mathrm{~cm}) ; 6$ - Apply 1 coat of adhesive (15 s);7- Light cure $(20 \mathrm{~s}$ at $600 \mathrm{~mW} /$ $\mathrm{cm}^{2}$ )8-Apply one coat of adhesive (15 s);9- Light cure (20 $\mathrm{s}$ at $600 \mathrm{~mW} / \mathrm{cm}^{2}$ )

1- Acid etching (15 s);

2- Wash (15 s);

3- Dry (5 s at $10 \mathrm{~cm})$;

4- Apply one coat of sealant;

5- Light cure (20 s at $\left.600 \mathrm{~mW} / \mathrm{cm}^{2}\right)$

Apply composite in $2 \mathrm{~mm}$-thick increments and light cure (30 s)

$37 \% \mathrm{H}_{3} \mathrm{PO}_{4}(15 \mathrm{~s}) ;$ Wash (15 s); Air stream (10 s); Adhesive (2 coats); Air stream (10 s); Light cure $(10 \mathrm{~s}$ at $600 \mathrm{~mW} /$ $\left.\mathrm{cm}^{2}\right)$; Composite restoration

HEMA (2-hydroxyethylmethacrylate); UDMA (urethane dimethacrylate); TEGDMA (triethyleneglycol dimethacrylate); Bis-GMA (bisphenol A-glycidyl methacrylate); Bis-EMA (bisphenol ethyl methacrylate). 
high-precision cutting machine (Isomet 1000; Buehler Ltd, Lake Bluff, IL, USA). Perpendicular sections to the bonding interface in the mesiodistal direction were obtained, resulting in 0.9-mm-thick slices. Each slice was individually positioned and the flattest area of the occlusal interface was delimited for a second section, in the buccolingual direction, producing stick-shaped samples of $0.8 \mathrm{~mm}^{2}$.

The stick samples of each tooth were allocated into two groups (24-hour and six-month) and subjected to tension at $0.5 \mathrm{~mm} / \mathrm{min}$ in a universal testing machine (EMIC DL2000; São José dos Pinhais, PR, Brazil). Bond strength analysis was performed $24 \mathrm{~h}$ after application of the sealants ${ }^{12-13}$. After 6 months of water storage in distilled water at $37{ }^{\circ} \mathrm{C}$, the procedure was repeated and fragments were observed in an optical microscope (Bel MicroImage Analyser; Bel Photonics do Brasil Ltda, Osasco, São Paulo, Brazil) at 40x magnification. Fractures were classified as cohesive (enamel or composite), adhesive (interface), or mixed (presence of composite and/or enamel in the same fragment). Percentage of fracture modes and specimens fractured before testing, were recorded for all groups ${ }^{13}$. For statistical purposes, the tooth was considered as the experimental unit. Average values of bond strength $(\mathrm{MPa})$ of each group were analyzed by repeated measures ANOVA and Tukey's test $(\alpha=5 \%)$.

\section{Results}

Table 2 presents the microtensile bond strength after 6 months for all experimental groups. There were differences for dentition $(p=0.0097)$, dental sealant $(p=0.0019)$ and time $(p=0.0001)$. At $24 \mathrm{~h}$, the highest bond strength was observed for OptiBond FL in deciduous and permanent dentition, similar to Fluroshield in deciduous and permanent dentitions. After 6 months of water storage, the bond strength of OptiBond FL decreased in deciduous teeth similar to Fluroshield in permanent teeth. The lowest bond strength after this time was observed for Fluroshield in deciduous teeth. The bond strength of OptiBond FL did not decrease in permanent dentition after 6 months.

The Repeated Measures ANOVA did not indicate statistically significant differences for the three-way interaction effect (statistics $F_{d f(1: 12)}=2.74 ;$ p-value $=0.1240$ $>0.05$ ), and both for two-way effects: tooth and time (statistic $F_{\text {df (1:12) }}=3.20 ;$ p-value $\left.=0.0990>0.05\right)$ and for tooth and sealant $\left(\right.$ statistic $F_{\text {df (1:12) }}=0.05 ;$ p-value $\left.=0.8197>0.05\right)$.

Table 3 presents the observed fracture types. In all groups mixed fractures were prevalent over "other fractures" (where cohesive fractures in enamel, cohesive fractures in resin and premature failures were grouped because their percentages were lower than those of mixed fractures and were not used for statistical purposes). Adhesive fractures were not observed.

\section{Discussion}

The hypothesis of this study was rejected since the bonding durability of resin materials to enamel varied among the experimental conditions. The American Academy of Pediatric Dentistry ${ }^{19}$ recognizes that the application and continued maintenance of sealants can prevent pit and fissure caries in children's teeth. However, enamel adherence to the occlusal surface of primary teeth via adhesive systems has not yet been widely investigated ${ }^{12-13,17}$. A few studies have tested the effectiveness and durability of hydrophilic adhesive sealants in caries prevention ${ }^{11,20}$.

In 2006, Feigal and Donly ${ }^{21}$ reported a modified technique using a bonding primer and adhesive layer between the etched enamel and the sealant. The technique overcame successfully the negative effects of salivary contamination that often occurs in pediatric patients. It has been shown ${ }^{22}$ that the inclusion of the primer and adhesive layer improves the bonding to the enamel and minimizes microleakage under conditions of saliva contamination.

Table 2 - Mean (standard deviation) of bond strength in MPa in the groups

\begin{tabular}{|c|c|c|c|c|}
\hline \multirow{3}{*}{ Material } & \multicolumn{4}{|c|}{ Type of dentition/ time } \\
\hline & \multicolumn{2}{|c|}{ Deciduous } & \multicolumn{2}{|c|}{ Permanent } \\
\hline & $24 \mathrm{~h}$ & 6 months & $24 \mathrm{~h}$ & 6 months \\
\hline OptiBond FL & $31.35(0.40) \mathrm{A}$ & 16.91(1.20)B,C & $29.89(2.50) A$ & 24.42(5.19)AB \\
\hline Fluroshield & 24.62(2.77)AB & $14.73(1.84) \mathrm{C}$ & 27.97(3.61)A & $18.42(5.60) B C$ \\
\hline
\end{tabular}

Different letters indicate statistically significant difference $(p<0.05)$.

Table 3 - Distribution of fractures in the sticks of the experimental groups according to the type of dentition and time.

\begin{tabular}{|c|c|c|c|c|}
\hline \multirow{4}{*}{ Material } & \multicolumn{4}{|c|}{ Fracture pattern } \\
\hline & \multicolumn{2}{|c|}{ Deciduous } & \multicolumn{2}{|c|}{ Permanent } \\
\hline & $24 \mathrm{~h}$ & 6 months & $24 \mathrm{~h}$ & 6 months \\
\hline & (M/OP) & (M/OP) & (M/OP) & (M/OP) \\
\hline \multirow[t]{2}{*}{ OptiBond FL } & $(25 / 0)(100 / 0 \%)$ & $(15 / 10)$ & $(40 / 0)$ & $(27 / 13)$ \\
\hline & & $(60 / 40 \%)$ & $(100 / 0 \%)$ & $(67,5 / 32.5 \%)$ \\
\hline \multirow[t]{2}{*}{ Fluroshield } & $(20 / 0)(100 / 0 \%)$ & $(13 / 7)$ & $(22 / 0)$ & $(17 / 5)$ \\
\hline & & (65/35\%) & $(100 / 0 \%)$ & (77/23\%) \\
\hline
\end{tabular}

M-fracture Mixed; OP-other patterns (sum of cohesive resin, of cohesive enamel and, premature failures). 
This study aimed at analyzing the bonding durability of a hydrophobic sealant and an hydrophilic adhesive system on deciduous and permanent teeth along 6 months of water storage. Results at $24 \mathrm{~h}$ indicated high bond strength for OptiBond FL in deciduous and permanent teeth, which was similar to Fluroshield in both dentitions. These similarities may be partially explained by the filler content in both pit and fissure sealants, which is around 50\% in Fluroshield and $48 \%$ in the hydrophobic adhesive OptiBond $\mathrm{FL}^{13,17}$. Both dental sealants were applied to deciduous and permanent teeth following acid etching with phosphoric acid, so the micro-retentive surfaces to be filled with dental sealants may have been produced similarly in both dentitions and materials.

According to Pashley et al. 201123, adhesive systems that present acid, primer and hydrophobic adhesive in separate bottles and no solvent in adhesive are superior in terms of adhesion durability, since there is no influence of hydrophilic compounds on the degree of conversion of the polymer, and OptiBond FL is an example of this. This may explain why the bond strength of OptiBond FL did not decrease from 24 $\mathrm{h}$ to 6 months in the permanent dentition. Other researches have described the good performance for OptiBond $\mathrm{FL}^{13,17}$ in deciduous teeth. The advantage of using a hydrophilic adhesive system as sealant was emphasized ${ }^{13,17}$ by minimizing the risk of saliva contamination in pediatric dentistry ${ }^{12-13}$. This was emphasized by Romito et al. ${ }^{13,17}$, who obtained the worst results for hydrophobic sealant under saliva contamination in their in vitro results. Fluroshield is a hydrophobic sealant that may have undergone the same consequences under saliva contamination as tested in the present in vitro study.

Regarding the type of dentition, differences may be considered in terms of the aprismatic enamel layer, which is thicker in deciduous than in permanent teeth ${ }^{2-3}$ and more resistant to acid etching ${ }^{24}$. This could explain the similar decrease of bond strengths of OptiBond FL and Fluroshield in deciduous teeth after 6 months, considering the pit-andfissure sealant itself in the present study. On the other hand, the type of dentition did not influence the bond strength of Fluroshield over time, because it decreased similarly in deciduous and permanent teeth.

After 6 months of water storage, decreased bonding was also observed for OptiBond FL on deciduous teeth, similar to Fluroshield on permanent teeth. The bond strength of OptiBond FL in permanent teeth was also similar to Fluroshield in deciduous teeth after $24 \mathrm{~h}$. Apart from the differences related to the type of dentition, due to the aprismatic enamel layer and composition of materials, these findings may also be explained by the method used to evaluate the bond strength in enamel, the microtensile bond strength. As enamel is a friable substrate, the diamond disc to obtain the specimens produces flaws that should be minimized by using diamond wires as previously described ${ }^{25}$. This may be considered a limitation of the present study, but the choice for using microtensile to test the bond to enamel was supported by a systematic review that revealed that this methodology is advantageous over micro-shear and other bond strength tests for allowing comparison of the adhesion of in vitro results to that of Class $\mathrm{V}$ in clinical circumstances ${ }^{26}$. In addition to the differences observed among the experimental groups, the fracture analysis of dental specimens showed prevalence of mixed fractures in all groups, which means that the enamel/interface was tested in tensile as expected. They were considered for statistical purposes, and the percentage of other fracture types in all groups was low.

Lemos et al. ${ }^{27}$ reported that the assiduity factor positively influences the dental caries prevalence. It is therefore essential, in every dental visit, not only the choice of the dental sealant, but to assess the risk of the child to develop dental caries. A recent literature review ${ }^{28}$ showed that the application of sealants to the occlusal surfaces of permanent molars in children and adolescents reduced caries onset up to 48 months when compared with no sealant application. It is important to consider this modality of treatment for children, regarding the maintenance of the masticatory function ${ }^{29}$ by means of a caries-free dentition. Other studies should be encouraged to evaluate clinically the use of different types of materials as pit-and-fissure sealants.

\section{References}

1. GwinnettAJ, Garcia-Godoy F. Effect of etching time and acid concentration on resin shear bond strength to primary tooth enamel. Am J Dent. 1992; 5: 237-9.

2. Mortimer KV. The relationship of deciduous enamel structure of dental disease. Caries Res. 1970; 4: 206-23.

3. Fava M, Watanabe I, Moraes FF, Costa LRRS. Prismless enamel in human non erupted deciduous molar teeth: Ascanning electron microscopic study. Rev Odontol Univ São Paulo. 1997; 11: 239-43.

4. Sonju Clasen $A B$, Ruyter IE. Quantitative determination of type A and type $B$ carbonate in human deciduous and permanent enamel by means of Fourier transform infrared spectrometry. Adv Dent Res. 1997; 11: 523-7.

5. Marquezan M, da Silveira BL, Burnett LH Jr, Rodrigues CR, Kramer PF. Microtensile bond strength of contemporary adhesives to primary enamel and dentin. J Clin Pediatr Dent. 2008; 32: 127-32.

6. Wang L, Sakai VT, Kawai ES, Buzalaf MA, Atta MT. Effect of adhesive systems associated with resin-modified glass ionomer cements. J Oral Rehabil. 2006; 33: 110-6.

7. Mattos-Graner RO, Rontani RM, Gavião MB, Bocatto HA. Caries prevalence in 6-36-month-old Brazilian children. Community Dent Health. 1996; 13: 96-8.

8. Amaral RC, Batista MJ, Meirelles MPMR, Cypriano S, Sousa MLR. Dental caries trends among preschool children in Indaiatuba, SP, Brazil. Braz J Oral Sci. 2014; 13: 1-5.

9. Hicks MJ, Flaitz CM. Caries formation in vitro around a fluoride-releasing pit and fissure sealant in primary teeth. ASDC J Dent Child. 1998; 65: 161-8.

10. Feldens EG, Feldens CA, Araujo FB, Souza MA. Invasive technique of pit and fissure sealants in primary molars: a SEM study. J Clin Pediatr Dent. 1994; 18: 187-90.

11. Grande RHM, LimaACP, Rodrigues Filho LE, Witzel MF. Clinical evaluation of an adhesive used as a fissure sealant. Am J Dent. 2000; 13: 167-70.

12. Ramires-Romito AC, Reis A, Loguercio AD, Goes MF, Grande RH. Micro-tensile bond strength of adhesive systems applied on occlusal primary enamel. J Clin Pediatr Dent. 2004; 28: 333-8.

13. Ramires-Romito AC, Reis A, Loguercio AD, Hipólito VD, de Goes MF, Singer JM, et al. Microtensile bond strength of sealant and adhesive systems applied to occlusal primary enamel. Am J Dent. 2007; 20: 114-20.

14. Vargas CM, Casper JS, Altema-Johnson D, Kolasny CR. Oral health 
trends in Maryland. J Public Health Dent. 2012; 72: S18-22.

15. Kanehira M, Finger WJ, Ishihata H, Hoffmann M, Manabe A, Shimauchi A et al. Rationale behind the design and comparative evaluation of an all-inone self-etch model adhesive. J Dent. 2009; 37: 432-9.

16. Gomes GB, Almeida LH, Oliveira AS, Moraes RR. Influence of water concentration on the etching aggressiveness of self-etch primers to ground primary enamel. Pediatr Dent. 2012; 34: 226-30.

17. Lemos LVFM, Felizardo KR, Myaki SI, Lopes MB, Moura SK. Bond strength and morphology of resin materials applied to the occlusal surface of primary molars. Int J Paediatr Dent. 2012; 22: 435-41.

18. Simonsen RJ. Pit and fissure sealant: review of the literature. Pediatr Dent. 2002; 24: 393-414.

19. American Academy of Pediatric Dentistry. Policy on third-party reimbursement of fees related to dental sealants. Pediatr Dent. 2012; 33: 85-6.

20. Grande RH, Ballester R, Singer JM, Santos JF. Microleakage of a universal adhesive used as a fissure sealant. Am J Dent. 1998; 11: 109-13.

21. Feigal RJ, Donly KJ. The use of pit and fissure sealants. Pediatr Dent. 2006; 28: 143-50; discussion 192-8.

22. Hebling J, Feigal RJ. Use of one-bottle adhesive as an intermediate bonding layer to reduce sealant microleakage on saliva-contaminated enamel. Am J Dent. 2000; 13: 187-91.

23. Pashley DH, Tay F, Breschi L, Tjasderhane L, Carvalho RM, Carrilho $M$ et al. State of the art of etch and rinse adhesives. Dent Mater. 2011; 27: 1-16.

24. Daronch M, De Goes MF, Grande RH, Chan DC. Antibacterial and conventional self-etcing primer system: morphological evaluation of intact primary enamel. J Clin Pediatr Dent. 2007; 27: 251-6.

25. Sadek FT, Monticelli F, Muench A, Ferrari M, Cardoso PE. A novel method to obtain microtensile specimens minimizing the cutting flaws. J Biomed Mater Res B Appl Biomater. 2006; 78: 7-14.

26. Heintze S. Systematic reviews I. The correlation between laboratory tests on marginal quality and bond strength. II. The correlation between marginal quality and clinical outcomes. J Adhes Dent. 2007; 9: 77-106.

27. Lemos LVFM, Barata TJE, Myaki SI, Walter LRF. Dentistry for babies: caries experience vs. assiduity in clinical care. Braz J Oral Sci. 2012; 11: 486-91.

28. Ahovuo-Saloranta A, Forss H, Walsh T, Hiiri A, Nordblad A, Makela M et al. Sealants for preventing dental decay in the permanent teeth. Cochrane Database Syst Rev. 2013; 3: CD001830.

29. Motta LJ, Santos JG, Alfaya TA, Guedes CC, Godoy CHL, Busadori SK. Clinical status of permanent first molars in children aged seven to ten years in a Brazilian rural community. Braz J Oral Sci. 2012; 11: 475-80. 